\title{
Propuesta metodológica para el desarrollo de productos electrónicos en Colombia
}

\author{
Robinson Castillo \\ Centro de Electricidad Electrónica y Telecomunicaciones, Servicio Nacional de Aprendizaje SENA, Grupo de \\ Investigación del CEET - SENA (GICS), Av. Carrera 30 \# 17B-25-Sur, Bogotá D.C., Colombia. \\ (Correo-e: rcastillo48@misena.edu.co)
}

Recibido May. 11, 2020; Aceptado Jul. 10, 2020; Versión final Sep. 10, 2020, Publicado Dic. 2020

\begin{abstract}
Resumen
Este trabajo presenta una propuesta metodológica para el desarrollo de productos electrónicos. La metodología corresponde a una adaptación de modelos de referencia para el desarrollo de productos electrónicos de empresas de base tecnológica, ajustado a la realidad de la industria electrónica colombiana y sus procesos de desarrollo de producto. La propuesta incluye cinco fases principales interrelacionadas: diagnóstico inicial, especificación del sistema, desarrollo del sistema, validación del sistema y gestión técnica. En la fase de validación del sistema, la realización de ensayos de seguridad eléctrica y ensayos de compatibilidad electromagnética básicos (inmunidad a perturbaciones conducidas y emisiones radiadas) se incluyen como principal elemento diferenciador. El trabajo presentado también incorpora una fase de diagnóstico inicial del sistema, dispositivo o producto, con el objetivo de obtener una caracterización inicial y un estado actual de funcionamiento; esta etapa ha sido contemplada para dar respuesta a la realidad de la industria electrónica colombiana.
\end{abstract}

Palabras clave: desarrollo de producto; sistemas en ingeniería; productos electrónicos

\section{Methodological proposal for electronic product development in Colombia}

\begin{abstract}
This work describes a methodological proposal for electronic product development. The proposed methodology corresponds to an adaptation of reference models for electronic product development into technology-based companies, in alignment to the reality of the Colombian electronics industry and its electronic product development process. The proposal includes five main interrelated phases: 1) initial system diagnosis, 2) system specification, 3) system development, 4) system validation, and 5) technical management. In the system validation phase, the performance of electrical safety tests and basic electromagnetic compatibility tests (immunity to conducted disturbances and radiated emissions) are included as the main innovative items. The present work also incorporates a phase for initial diagnosis of the system, device, or product in order to obtain the starting characterization and the actual working state. This phase is assessed in response to the needs of the Colombian electronic industry.
\end{abstract}




\section{INTRODUCCIÓN}

El Servicio Nacional de Aprendizaje - SENA, es un "establecimiento público de orden nacional del Estado Colombiano, encargado de ofrecer y ejecutar la formación profesional integral, para la incorporación y el desarrollo de las personas en actividades productivas que contribuyan al desarrollo social, económico y tecnológico de Colombia". En su infraestructura, el SENA cuenta con diferentes Centros de formación distribuidos en las diferentes regiones de Colombia. El Centro de Electricidad, Electrónica y Telecomunicaciones - CEET, es uno de ellos y es el quinto Centro de formación más grande del SENA, se ubica en el Distrito Capital. Una revisión de la experiencia y competencias de los instructores encargados de impartir la formación profesional vinculados en el área de Electrónica del CEET, evidenció fortalezas hacia el mantenimiento de equipos electrónicos, pero debilidades y bajas capacidades técnicas en el diseño y desarrollo de productos electrónicos, lo cual, dificulta al CEET fortalecer talento humano interno y externo con capacidades en desarrollo y producción de equipos electro electrónicos acorde con necesidades de la industria de dicho sector tanto en el Distrito Capital, como de la nación.

Considerando resultados de proyectos financiados por el Departamento Administrativo de Ciencia, Tecnología e Innovación - COLCIENCIAS, como el proyecto de "Prospectiva Tecnológica de la Industria Electro Electrónica de Bogotá y Cundinamarca", del cual se evidencia, específicamente en el apartado de innovación, la necesidad de fomentar la implementación de metodologías estructuradas de desarrollo de producto electro electrónico (Castillo et al., 2015), el CEET identificó la necesidad de fortalecer y generar capacidades técnicas en el diseño y desarrollo de productos electrónicos para repotenciar y/o crear nuevos productos mediante el diseño de una propuesta metodológica para tal fin, partiendo inicialmente de las necesidades y particularidades identificadas en este mismo Centro, y comunes en la industria electrónica colombiana.

El rápido progreso de la industria electrónica genera una evolución en los métodos que se adoptan para el desarrollo de sus productos. Existen metodologías para el desarrollo de sistemas de ingeniería y productos electrónicos (Aranguren et al., 2015; Mcharek et al., 2019; Chang y Perng, 2008), pero también técnicas ágiles para satisfacer las necesidades activas de las empresas y para una mejor comprensión del desarrollo de aplicaciones de software (Mesa et al., 2018).

Esta propuesta metodológica se ha generado a partir de la revisión de diferentes modelos para el desarrollo de sistemas en ingeniería, también de los aspectos contenidos en las Normas Sectoriales de Competencias Laborales - NSCL, de la asesoría recibida por el CEET de empresas del sector industrial colombiano que desarrollan soluciones de ingeniería a la medida tomando como base metodológica el estándar ANSI/EIA 632 (Electronic Industries Alliance, 1999), y de la identificación de capacidades tecnológicas y tecnología ubicada en este Centro de Formación Profesional. Se propone una metodología de cinco fases articuladas: diagnóstico inicial del producto, especificación del sistema, desarrollo del sistema, validación del sistema y gestión técnica. Para el caso de Colombia, la propuesta incorpora la realización de ensayos o pruebas orientadas a garantizar la seguridad eléctrica del producto electrónico y la realización de ensayos de Compatibilidad Electromagnética básicos (Heirman, 2016), ensayos que los desarrolladores de producto electrónico no aplican o no se realizan en el país.

\section{METODOLOGÍA}

Como se muestra en la figura 1, el proceso seguido para el establecimiento de la propuesta metodológica para el desarrollo de producto electrónico ha constado de las siguientes fases: Identificación de los principales modelos y/o metodologías existentes, identificación de sus características y procesos principales, identificación de las particularidades del CEET en lo relacionado a desarrollo de producto electrónico, análisis y comparación de todos los elementos identificados, el establecimiento de la propuesta metodológica para el desarrollo de producto electrónico y finalmente una validación de la misma mediante un caso de aplicación. A partir de los modelos identificados se consolida una representación de los procesos y datos relacionados al desarrollo de producto, a manera de ilustración, de acuerdo a ANSI/EIA 632 (Electronic Industries Alliance, 1999), se identifican los siguientes estados para el proceso de desarrollo de sistemas en ingeniería: necesidades (N), Requerimientos (R), Representación Lógica (L) y Representación Física (P), modelo NRLP (Mirdamadi et al., 2018).

Internamente, el CEET ha realizado un diagnóstico que le ha permitido establecer tanto capacidades, como tecnología disponible para los procesos de formación e investigación, identificando particularidades y potenciales ventajas aplicables en un proceso de desarrollo de producto electrónico. También se ha realizado la transferencia en el modelo de desarrollo tecnológico, de gestión de la tecnología e innovación en su departamento de I+D+i, implementado por una empresa del sector electrónico colombiano, enfocada en el desarrollo de soluciones de ingeniería a la medida. 


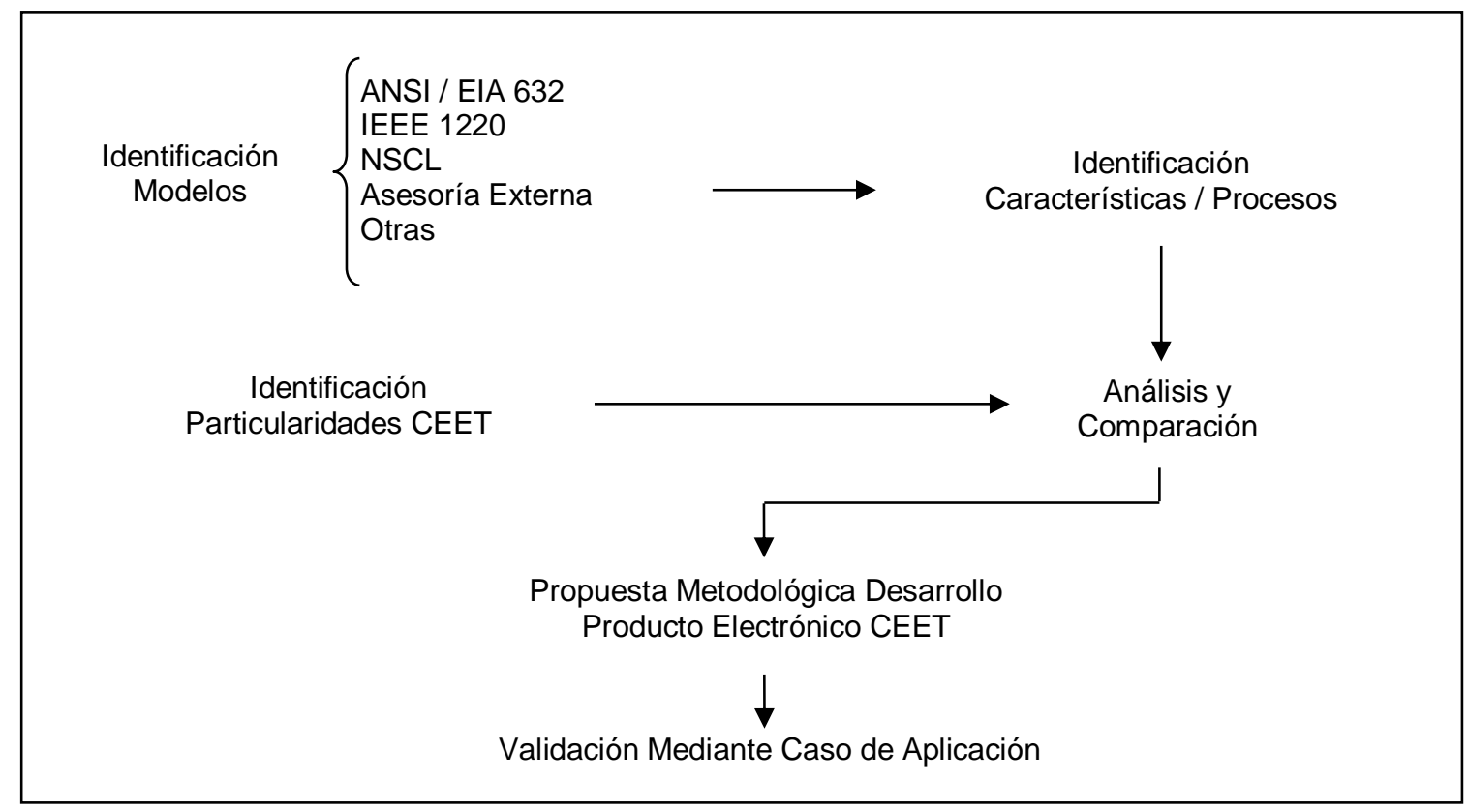

Fig. 1: Metodología seguida para el establecimiento de la propuesta metodológica

Una vez establecidos estos elementos, se ha realizado una clasificación y priorización de procesos y actividades, llegando a una generalización de pasos y taxonomía básica del proceso, basadas en los estándares y modelos revisados, incluyendo los elementos posibles diferenciadores en cuanto a tecnología identificados previamente en el CEET. Los anteriores elementos han permitido establecer la propuesta metodológica para el desarrollo de producto electrónico.

\section{Principales Modelos}

Existen diferentes maneras de representar la línea de desarrollo de un producto electrónico, desde el instante en el cual la primera idea es concebida, el trabajo realizado para lograr dicho desarrollo, hasta el momento en el cual el producto se encuentra a la venta. El informe CDIO, emitido por el Instituto Tecnológico de Massachusetts - MIT organiza el desarrollo y la enseñanza de la electrónica en cuatro etapas: concepción, diseño, implementación y operación (Aranguren et al., 2015). Estas etapas básicas, se identifican en diferentes estándares para el desarrollo de sistemas de ingeniería o productos.

EI ANSI / EIA 632 (Electronic Industries Alliance, 1999) no especifica el cómo implementar los requerimientos que especifican el diseño del sistema, los métodos y herramientas aplicables deben ser seleccionadas por quien desarrolle el sistema; tampoco hace énfasis en formatos, estructura o medios de documentación; establece cinco grandes fases para el desarrollo de sistemas en ingeniería (figura 2): adquisición y suministro, gestión técnica, diseño del sistema, realización del producto y evaluación técnica.

ANSI/EIA 632 también define 13 procesos que contienen un total de 33 requisitos que se utilizan en la ingeniería de un sistema. Puede aplicarse al proceso de desarrollo de cualquier producto. Un concepto clave en el ANSI/EIA 632 es el de un modelo de sistema orientado en torno a "Building Blocks". Cada bloque es un sistema en sí mismo y, por lo tanto, consta de los productos finales, definidos por la necesidad del cliente y realizan las funciones requeridas por este mismo, además de los productos y procesos que pueden denominarse productos habilitantes, los cuales son necesarios para desarrollar, probar, implementar, utilizar, respaldar y retirar esos productos.

IEEE 1220 (IEEE Computer Society, 2005), "Standard for Application and Management of the Systems Engineering Process" establece un modelo de proceso de sistemas y describe las actividades en relación con su esfuerzo total de ingeniería para lograr un resultado determinado (es decir, un producto o servicio). El modelo de proceso de sistemas ilustra la secuencia y/o la interacción entre varias actividades del proyecto desde la creación hasta la eliminación del producto/servicio. El modelo de proceso de ingeniería descrito en IEEE 1220 incluye: (1) Análisis de requerimientos; (2) Validación de requerimientos; (3) Análisis funcional; (4) Verificación Funcional; (5) Síntesis; y (6) Verificación Física. Estos procesos están vinculados entre sí a través de procesos de control que consisten en la gestión de datos; Gestión de la configuración; Gestión de interfaz; Gestión de riesgos y mediciones del progreso basadas en el rendimiento. 


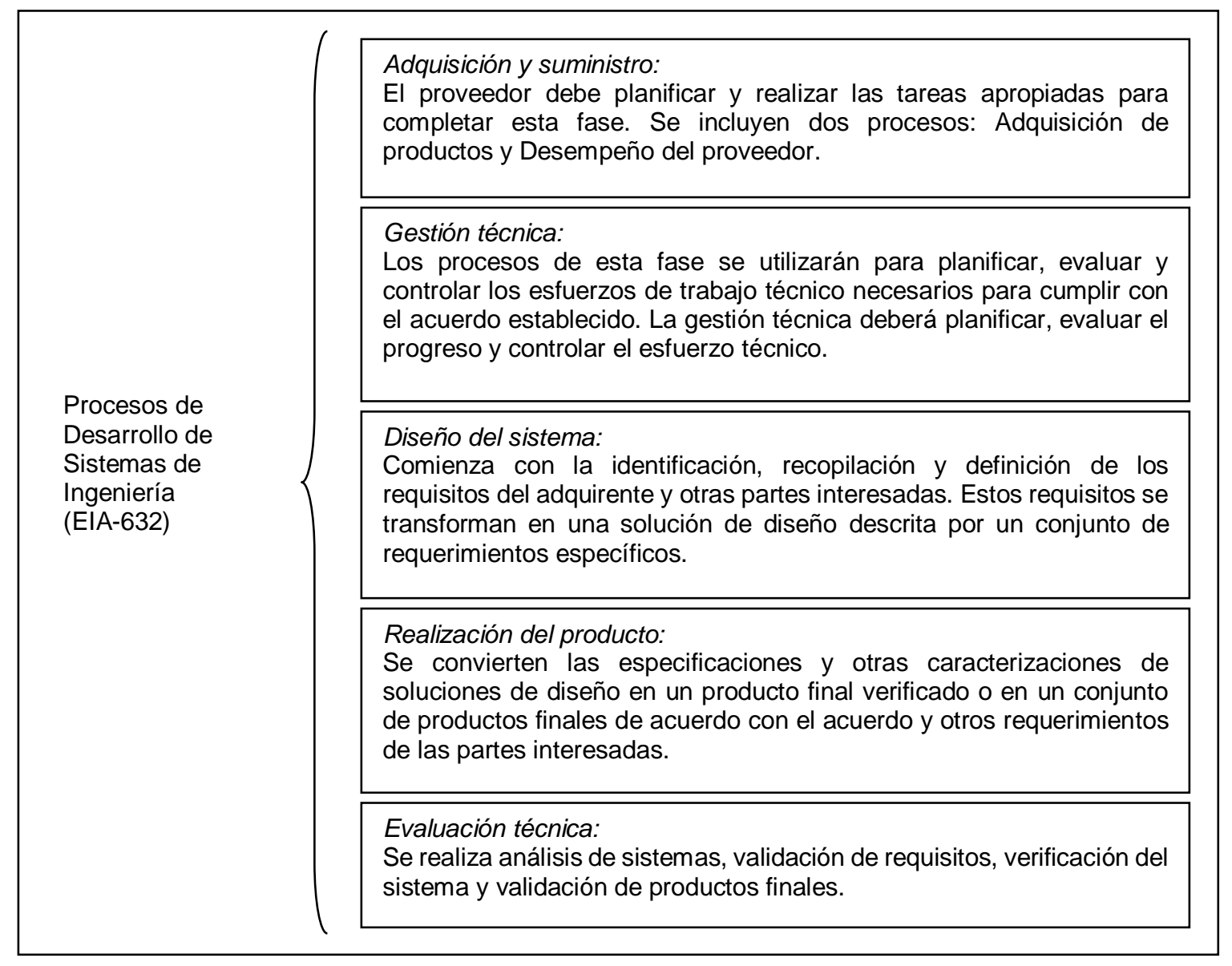

Fig. 2: Fases Desarrollo de Sistemas de Ingeniería EIA 632

El modelo de desarrollo de productos STAR es un derivado de los procesos técnicos de la norma ISO 15288: 2008, que es lo suficientemente reducido para facilitar el control a nivel de diseño de los productos electrónicos por parte de las organizaciones adoptantes, mientras que la implementación puede ser subcontratada. Comprende siete procesos: (1) Definición de requerimientos; (2) Análisis de requerimientos; (3) Partición del sistema; (4) Implementación; (5) Abastecimiento; (6) Validación; (7) Integración. En cualquier momento dado en un proyecto de desarrollo de productos, se dice que el estado del proyecto se encuentra en uno de estos procesos (Salgado et al., 2014). Otros modelos, algunos usados para el desarrollo de software o con enfoques basado en el riesgo para el desarrollo de productos o sistemas, o modelos que incluyen iteraciones y que se desarrollan varias fases, como modelo cascada, espiral, Vee, SIMILAR ó STAR (STV+ANGLIA RUSKIN) (Aranguren et al., 2015; Chang y Perng, 2008; Cooper, 2019; Mcharek et al., 2019) hacen parte de los principales modelos identificados en relación con metodologías para el desarrollo de sistemas en Ingeniería o productos.

De acuerdo al trabajo titulado " Modelo conceptual para el desarrollo de producto en el área de "Electrónica" para la Universidad Distrital Francisco José de Caldas" se define el proceso de Desarrollo de Producto como la secuencia de pasos o actividades que realiza una organización para concebir, diseñar y comercializar un producto, considerando que en el caso de productos tecnológicos, su desarrollo se consigue mediante la participación de equipos multidisciplinarios en las siguientes etapas genéricas:

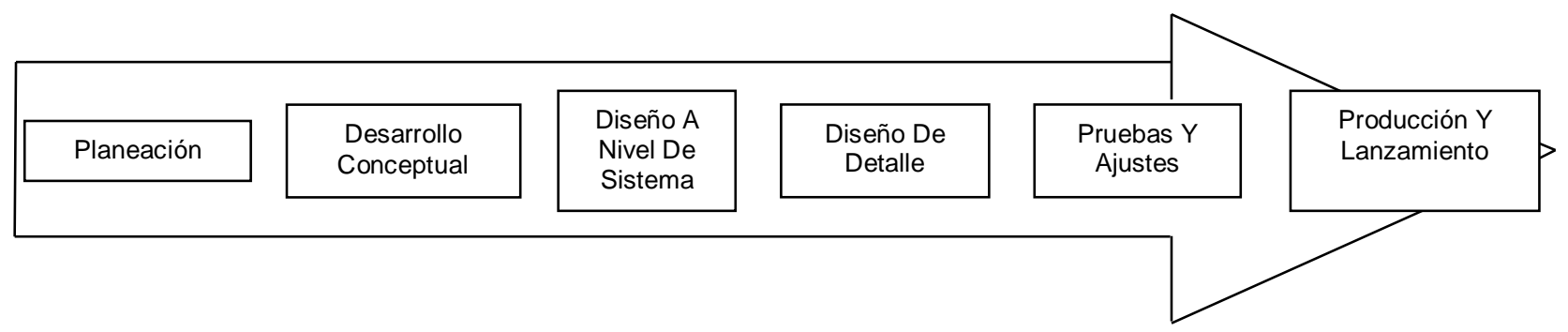

Fig. 3: Etapas genéricas en el proceso de desarrollo de producto 
Muchos casos, en las organizaciones estos pasos y actividades son intelectuales más que físicos. Algunas definen y siguen un proceso de desarrollo preciso y detallado, mientras que otras organizaciones en ocasiones no logran describir sus procesos. Los modelos de desarrollo de producto podrían agruparse en dos grandes categorías: i) Modelos industriales, ampliamente conocidos y utilizados en todo el mundo por industrias de diferente tipo; y ii) Modelos académicos, construcciones teóricas que en la mayoría de los casos nacen al interior de las Universidades, centros de desarrollo, centros de investigación y constituyen propuestas académicas que luego de su validación son acogidas por la industria y usuarios externos.

\section{RESULTADOS Y DISCUSIÓN}

La mayoría de la literatura identificada acerca de Desarrollo de Nuevo Producto se centra en las actividades de grandes empresas conocidas o dentro del contexto de economías bien desarrolladas, pero la literatura dentro de las pequeñas y medianas empresas ubicadas en países en desarrollo es más limitada (Salgado et al., 2014). La propuesta metodológica para el desarrollo de producto electrónico presentada, se deriva de los procesos técnicos contenidos en estándares existentes para el desarrollo de sistemas, procesos de desarrollo construidos a partir de la experiencia de entidades del entorno local dedicados al desarrollo de productos electrónicos como proyectos de desarrollo de soluciones a la medida, y de la identificación de necesidades y la dinámica de funcionamiento de la industria electrónica local; está orientada a sistemas convencionales, agregando características propias en el diseño y desarrollo del mismo (Zapata et al., 2011), enfocando los aportes metodológicos en la fase de validación.

Esta propuesta metodológica contempla cinco grandes fases articuladas, tomando como punto de partida la identificación de una necesidad u oportunidad, la cual pueda ser atendida por medio del desarrollo y materialización de un producto electrónico, estas fases son: el diagnóstico inicial del producto (cuando se trate de una adaptación de un diseño existente), la especificación del sistema, el desarrollo del sistema, la validación del sistema y de manera transversal, una gestión técnica del desarrollo, la propuesta metodológica se resume en la figura 4 .

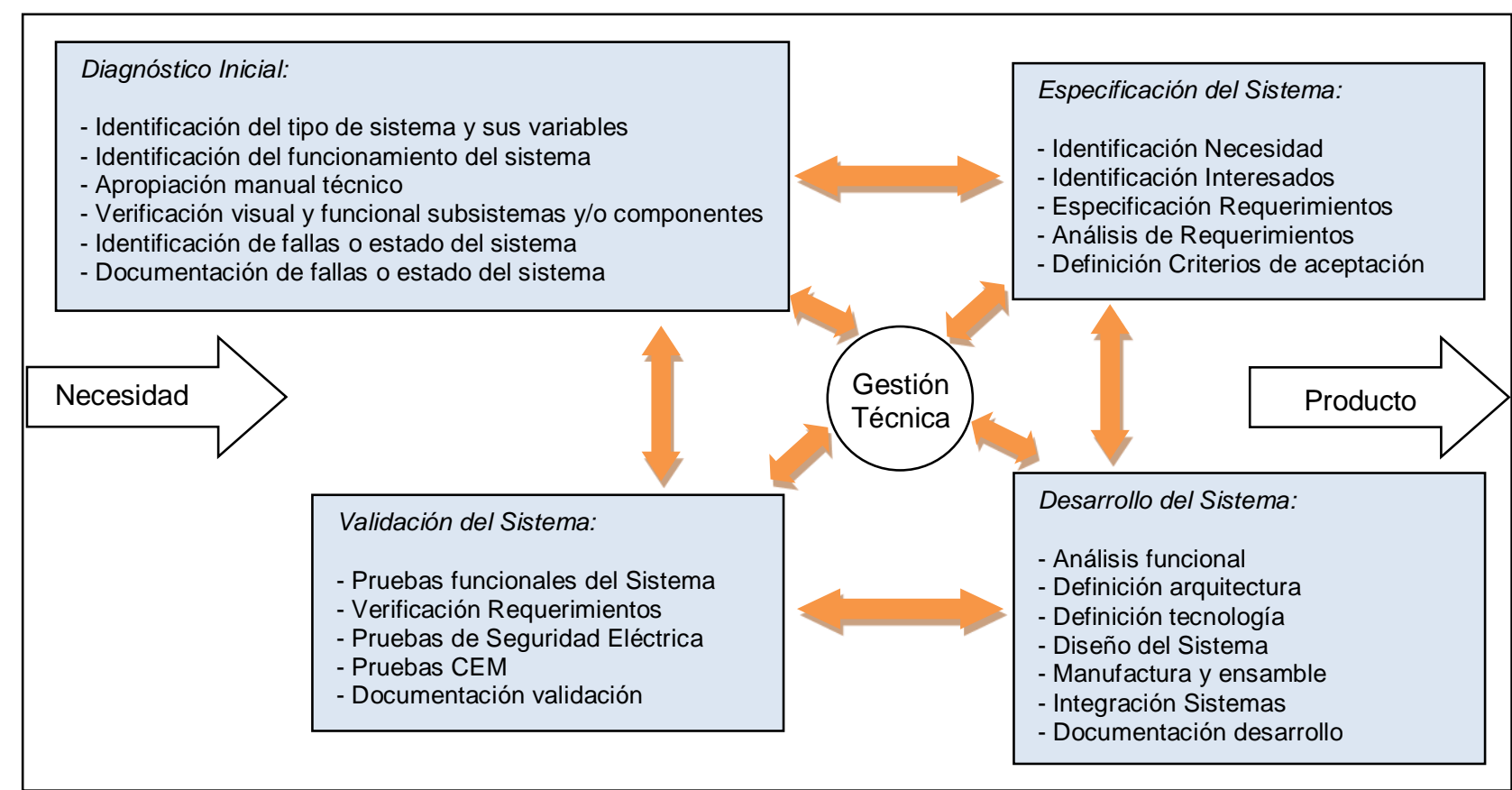

Fig. 4: Metodología Desarrollo de Producto Propuesta

Diagnóstico Inicial del sistema: Requerido si se trata de una adaptación o modificación de un diseño previo del sistema. Su objetivo es identificar el tipo de sistema (o equipo) y tipos de variables involucradas en su funcionamiento. Establecer los elementos, subsistemas y componentes conformantes para comprender su funcionamiento, identificar fallas y/o estado actual y obtener un diagnóstico del sistema o equipo para abordar la fase de especificación del sistema orientada al rediseño.

Especificación del sistema: Abarca el proceso de caracterización del sistema, el insumo de entrada corresponde a una necesidad u oportunidad, a partir de este punto se consolida la identificación de la misma 
atendiendo a las razones, importancia y desarrollo que se abordará. En esta fase también deben identificarse los involucrados, así como sus interés y nivel de importancia e influencia durante el proceso de desarrollo del producto. Para finalizar, en la especificación del sistema deben definirse los requerimientos funcionales y no funcionales, los técnicos, de desempeño y estratégicos, consolidando por medio de estos requisitos una representación muy cercana al producto final, así mismo, se establecen los criterios de aceptación y una línea base validación, elementos que permitirán posteriormente verificar el cumplimiento de los requerimientos definidos.

Desarrollo del sistema: Comprende todo lo relacionado al diseño y materialización del producto. A partir del establecimiento y análisis de los requerimientos, el desarrollo del sistema contempla un análisis funcional, la definición de una arquitectura, la determinación de la tecnología a incorporar en el producto, así como su representación lógica, a partir de este punto se aborda el diseño del sistema, esto es, el diseño electrónico detallado, esto incluye diagramas esquemáticos, software y/o firmware, simulaciones del sistema, diseño profesional de tarjeta de circuito impreso, diseño mecánico y demás partes o subsistemas especializados que se han identificado como constitutivos del producto electrónico a desarrollar. Durante esta fase es de gran importancia garantizar la relación y fluida comunicación entre las diferentes áreas involucradas, esto es, entre el diseñador electrónico, el diseñador de PCB, el diseñador mecánico, el desarrollador de firmware y demás integrantes de las diferentes áreas necesarias para el desarrollo del producto acorde a los requerimientos definidos para el producto.

El desarrollo del sistema también contempla la materialización, representación o implementación física del producto. Una vez establecido el diseño del sistema se han obtenidos los insumos para la manufactura del mismo, tarjetas electrónicas, encerramientos y partes especializadas. (Durante la validación metodológica aplicada, para la particularidad del CEET, se ha establecido un prototipado de PCB y la incorporación de impresión 3D para el desarrollo de encerramientos y algunas partes especializadas). Una vez fabricadas las tarjetas y partes especializadas puede iniciarse el ensamble de los componentes e integración del sistema, contemplando los respectivos protocolos de pruebas de ensamble establecidos a partir del diseño electrónico del sistema. La documentación de la manufactura y el ensamble también se encuentran contempladas en esta fase. Debe anotarse que la adquisición de componentes se encuentra contemplado dentro de las actividades de abastecimiento y suministro, las cuales se encuentran contempladas dentro de la fase transversal de Gestión Técnica.

Validación del sistema: Comprende todas las actividades de verificación y pruebas de funcionalidad a nivel de tarjetas de circuito impreso, partes especializadas, software, firmware, métricas de calidad y demás que garanticen el correcto funcionamiento del producto de acuerdo a los requerimientos definidos. Esta validación también contempla la validación de los requerimientos mediante la revisión y chequeo del cumplimiento de los respectivos criterios de aceptación de cada requerimiento, establecidos durante la etapa de Especificación del Sistema.

La propuesta metodológica presentada incorpora la realización de ensayos o pruebas orientadas a garantizar la seguridad eléctrica del producto electrónico y, la realización de ensayos de Compatibilidad Electromagnética básicos (inmunidad a perturbaciones conducidas y emisiones radiadas) de tal forma, que además de garantizar el cumplimiento de los requerimientos especificados para el sistema y la funcionalidad del mismo (Sauchelli et al., 2014), también se pueda asegurar que el sistema o dispositivo desarrollado es seguro desde el punto de vista de funcionalidad eléctrica y se encuentra dentro de los rangos establecidos por las diferentes directivas y normativas en cuanto a Compatibilidad Electromagnética durante su operación.

Gestión técnica: Fase transversal y de ejecución permanente durante todo el proceso de desarrollo del producto. Incluye la planeación, monitoreo y control de las actividades, la adquisición y suministro de materiales, insumos y/o componentes. La gestión técnica del desarrollo también incluye actividades de entrega y aceptación del producto, esto es, entrega del producto con requerimientos validados y aceptados, entrega de documentación generada durante todo el proceso de diseño y manuales y documento de aceptación de entrega del producto como soporte de cierre del proceso. La figura 4 muestra los procesos y actividades asociadas a cada fase de la propuesta metodológica para el desarrollo de productos electrónicos presentada, incluyendo un diagnóstico inicial del sistema y la realización de ensayos o pruebas orientadas a garantizar la seguridad eléctrica del producto electrónico y, la realización de ensayos de Compatibilidad Electromagnética básicos.

La aplicación de estos ensayos en la etapa de validación son elementos que no se identificaron en los estándares consultados en la etapa de identificación de modelos y sus características. Así mismo, para la industria electrónica colombiana, los ensayos de seguridad eléctrica y de compatibilidad electromagnética, son incipientes y no se encuentran formalizados y mucho menos estandarizados dentro de los procesos de desarrollo electrónico, en parte, bajo la argumentación de falta de laboratorios especializados para la 
aplicación de este tipo de pruebas (Castillo et al., 2015); sin embargo, su aplicación derivará en productos electrónicos de mayor calidad y competitividad a nivel global.

La metodología propuesta también se concibe acercándose a la realidad de algunos de los procesos realizados en la industria electrónica colombiana: la adaptación, rediseño y/o actualización de productos electrónicos. Esta fase es aplicada y válida cuando debe abordarse un ajuste o modificación de un sistema existente, es evidente que aunque no se trate de un nuevo dispositivo, es necesaria la aplicación de un proceso formal de desarrollo, entendido en esos casos como rediseño, pero que al final del mismo, derivará en un producto electrónico funcional.

La validación de la propuesta metodológica se ha realizado mediante un caso de aplicación, el cual ha consistido en la modificación al diseño de un Robot Industrial Educativo, un brazo robótico que cuenta con cinco ejes de movimiento más una pinza como actuador final, usado en actividades de formación profesional pero que se encontraba fuera de servicio.

En la fase de Diagnóstico Inicial al Robot industrial Educativo se le ha realizado una identificación de sus conexiones para analizar su funcionamiento. Se ha identificado cada subsistema, aislando los diferentes elementos defectuosos o dañados. Se evalúo la frecuencia de funcionamiento de cada encoder en función el voltaje en un rango de 6 VDC a 12 VDC, se identificó un funcionamiento en un rango de $345 \mathrm{~Hz}$ a $877 \mathrm{~Hz}$, siendo su valor medio $615,7 \mathrm{~Hz}$ con un voltaje aplicado de 9 VDC. Uno de los encoders del sistema evidenció fallo total. Se incluyó inversión de polaridad del voltaje para la medición de respuesta en frecuencia de cada dispositivo. De la etapa de diagnóstico, se ha determinado que el robot industrial educativo se encuentra en una funcionalidad aproximada del $45 \%$.

En la fase de Especificación del sistema se inició con la identificación de los interesados en el proyecto y los establecimientos de su nivel de importancia. Como principal especificación del sistema se evidenció que debían desarrollarse las tarjetas electrónicas que permitan realizar el control de los dispositivos, con conectividad a una estación de trabajo con un sistema operativo y conectividad de red entre las que se consideraron Wi-Fi, USB, Ethernet, MODBUS y CAN. Se debieron desarrollar los modelos tridimensionales de la estación de trabajo. La descripción de requerimientos especificó una descripción corta y general del sistema en su encabezado, y para cada requerimiento, un código identificador, tipo de requerimiento, descripción del requerimiento, función asociada, desempeño, prioridad y criterio de aceptación. El nuevo diseño del robot en este caso quedó definido mediante veintitrés (23) requerimientos funcionales y dieciséis (16) requerimientos no funcionales.

En la fase de Desarrollo del sistema se ha definido la arquitectura de la solución y la tecnología a incorporar. Se ha incorporado tecnología de montaje superficial, un sistema microcontrolado especializado en aplicaciones de control de motores, IloT, CAN multicanal y arquitectura RISC. Comunicación Wi-Fi, USB, Ethernet, MODBUS y CAN se han incluido. Una interfaz de usuario con tres niveles de usuario (administrador, instructor y aprendiz) también ha sido implementada. En la fase de Validación del sistema la etapa de control, de comunicaciones y todo el sistema incorporado en el Robot Industrial Educativo deberá someterse a los ensayos de Seguridad Eléctrica conforme a la norma IEC 61010-2-201:2018, Parte 2-201: Requisitos particulares para Equipos de Control. A los ensayos de Compatibilidad Electromagnética conforme a las normas IEC 61000-4-6:2014 Técnicas de ensayo y de medida. Inmunidad a las perturbaciones conducidas, inducidas por los campos de radiofrecuencia, y a los de la norma IEC 61000-4-20:2011 Técnicas de ensayo y de medida. Ensayos de emisión y de inmunidad en las guías de onda electromagnéticas transversales (TEM), esto de acuerdo a la metodología propuesta, específicamente considerando el aporte realizado a las metodologías de desarrollo, el cual se fundamenta en la incorporación de estos ensayos en los procesos de desarrollo de equipo electrónico, especialmente en Colombia.

\section{CONCLUSIONES}

Se ha presentado una propuesta metodológica enfocada en el desarrollo de producto electrónico partiendo de la identificación de los principales modelos para el desarrollo de productos y/o sistemas en ingeniería, una transferencia tecnológica recibida por parte de una empresa experimentada en diseño y desarrollo de soluciones de ingeniería a la medida y la identificación de capacidades del CEET. La metodología propuesta divide el desarrollo del producto en "planos de interrelación", cada plano o fase consiste en una abstracción de cada dominio de desarrollo, donde cada dominio de desarrollo representa una característica de la integración de las unidades que conforman el sistema y configuran al finalizar el producto.

La propuesta metodológica se basa en las etapas básicas de concebir, desarrollar y validar, incorporando como elemento innovador la realización de Ensayos de Seguridad Eléctrica conformes a la norma IEC aplicable según la tipología de producto y Ensayos básicos de Compatibilidad Electromagnética conforme a las normas IEC 
61000-4-6:2014 Técnicas de ensayo y de medida. Inmunidad a las perturbaciones conducidas, inducidas por los campos de radiofrecuencia, y a los de la norma IEC 61000-4-20:2011 Técnicas de ensayo y de medida. Ensayos de emisión y de inmunidad en las guías de onda electromagnéticas transversales (TEM), esto es inmunidad a perturbaciones conducidas y emisiones radiadas en la fase de validación.

Con este trabajo se pretende adaptar un modelo de referencia para el proceso de desarrollo de productos electrónicos de empresas de base tecnológica de pequeño y mediano tamaño, incorporando al modelo de referencia propuesto una necesidad manifiesta de la industria electrónica nacional, los ensayos básicos de Seguridad Eléctrica y Compatibilidad electromagnética, validación que no fue identificada en los modelos y estándares para el desarrollo de productos analizados.

El trabajo presentado también incorpora una fase de diagnóstico inicial del sistema, dispositivo o producto, con el objetivo de obtener una caracterización inicial y un estado actual de funcionamiento; esta etapa ha sido contemplada para dar respuesta a la realidad de la industria electrónica colombiana y sus procesos de desarrollo de producto, la cual, en varios casos se enfrenta a la adaptación, rediseño y/o actualización tecnológica de productos electrónicos.

\section{AGRADECIMIENTOS}

Este trabajo fue desarrollado por el Centro de Electricidad, Electrónica y Telecomunicaciones - CEET en el marco del proyecto "Innovación metodológica para el desarrollo de productos electrónicos en el Centro de Electricidad, Electrónica y Telecomunicaciones - CEET", el autor extiende su agradecimiento al Sistema de Investigación, Desarrollo Tecnológico e Innovación del SENA-SENNOVA, como financiadores para la realización de este trabajo y al Grupo de Investigación del CEET-SENA, como ejecutor del mismo. También agradece a la empresa KUSPYDE INGENIERÍA S.A.S por la realización de la transferencia tecnológica enfocada en su modelo de desarrollo tecnológico, de gestión de la tecnología e innovación en su departamento de l+D+i.

\section{REFERENCIAS}

Aranguren, G., Ortiz, J., y Gil-Garcia, J. M., From the Idea to the Product: An Academic Tour, https://doi.org/10.1109/RITA.2015.2486418, IEEE Revista Iberoamericana de Tecnologías del Aprendizaje, 10(4), 290295 (2015).

Castillo, R., Mayorquín, D., y Zuluaga, D., Prospectiva Tecnológica de la Industria Electro Electrónica de Bogotá y Cundinamarca, 1르. Ed., 158-169, Fondo de Publicaciones Universidad Sergio Arboleda, Bogotá D.C., Colombia (2015).

Chang, G., y Perng H., A review of systems engineering standards and processes, Journal of Biomechatronics Engineering, 1(1), 71-85 (2008).

Cooper, R. G., The drivers of success in new-product development, https://doi.org/10.1016/j.indmarman.2018.07.005, Industrial Marketing Management, 76, 36-47 (2019).

EIA-632: Electronic Industries Alliance, EIA STANDARD Processes for Engineering a System, 1-62, Arlington, USA (1999).

Heirman, D., EMC Standards Activity, IEEE Electromagnetic Compatibility Magazine, 5(1), 80-84, (2016).

IEEE Standard for Application and Management of the System Engineering Process, IEEE Computer Society, New York, USA (2005).

Mcharek, M., Hammadi, M., y otros tres autores, Collaborative design process and product knowledge methodology for mechatronic systems, https://doi.org/10.1016/j.compind.2018.12.008, Computers in Industry, 105, 213-228 (2019).

Mesa, J., Maury, H., y otros tres autores, A novel approach to include sustainability concepts in classical DFMA methodology for sheet metal enclosure devices, https://doi.org/10.1007/s00163-017-0265-4, Res Eng Design, 29, 227244 (2018).

Mirdamadi, S., Addouche, S. A., y Zolghadri, M., A Bayesian approach to model change propagation mechanisms, https://doi.org/10.1016/j.procir.2018.03.309, Procedia CIRP, 70, 1-6 (2018).

Salgado, E. G., Salomon, V. A. P., y otros dos autores, A reference model for the new product development in mediumsized technology-based electronics enterprises, https://doi.org/10.1109/TLA.2014.7014499, IEEE Latin America Transactions, 12(8), 1341-1348 (2014).

Sauchelli, V., Rabinovich, S., y otros tres autores, Celda Anecóica tipo GTEM, aspectos constructivos, Revista Tecnología y Ciencia, 12(2), 25-31 (2014).

Zapata, G., Cardillo, J., y Chacón, E., Aportes metodológicos para el diseño de sistemas de supervisión de procesos continuos, https://doi.org/10.4067/S0718-07642011000300012, Información Tecnológica, 22(3), 97-114 (2011). 